\title{
Sistemas de bombeo solar en Pachuca Hidalgo
}

\section{Solar pumping systems in Pachuca Hidalgo}

FLORES-RAMIREZ, Oscar $\dagger^{*}$, HUESCA-LAZCANO, Erick Eduardo, ROMERO-RODRIGUEZ, Gabriel y JIMENEZ-SOTO Ezequiel

Universidad Politécnica de Amozoc, Departamento de Ingeniería en Energía

ID $1^{\text {er }}$ Autor: Oscar, Flores-Ramírez / ORC ID: 0000-0001-9884-9499, Researcher ID Thomson: E-8242-2018, arXiv Author ID: 429567-444L3Z-UIWFRF, CVU CONACYT ID: 92914

ID $1^{\text {er }}$ Coautor: Erick Eduardo, Huesca-Lazcano / ORC ID: 0000-0002-0505-8442, Researcher ID Thomson: F-1162-2018, Open ID: 357590366000 , CVU CONACYT ID: 223342

ID $2^{\text {do }}$ Coautor: Gabriel, Romero-Rodríguez, / ORC ID: 0000-0002-5124-3016, Researcher ID Thomson: Y-2756-2018, CVU CONACYT ID: 299737

ID $3^{\text {er }}$ Coautor: Ezequiel, Jiménez-Soto / ORC ID: 0000-0001-6486-8122

DOI: $10.35429 /$ JSI.2019.12.3.17.27

Recibido 16 de Agosto 2019; Aceptado 10 Diciembre, 2019

\section{Resumen}

La extracción de agua de pozos para suministro en poblaciones rurales es una de las aplicaciones más rentables y novedosa de la energía solar fotovoltaica. El empleo de un tipo de bombas sumergibles específicas para aplicaciones con energía fotovoltaica, que funcionan a corriente continua/directa (CC/DC) generada directamente de los módulos fotovoltaicos, posibilita una instalación independiente de la red eléctrica con muy escasas necesidades de mantenimiento, en este proyecto se realizó el dimensionamiento, instalación y puesta en marcha de un sistema de bombeo autónomo con bomba sumergible alimentado por una instalación solar fotovoltaica en el municipio de Actopan Hidalgo en el estado de Pachuca, para implementar el proyecto se realizó primeramente un estudio climático de la zona para fundamentar la eficiencia del sistema de bombeo solar, en este proyecto se utilizaron 18 paneles de $150 \mathrm{~W}$, un controlador SubDrive Solar, una bomba Franklin Electric de 3 HP y un variador de frecuencia para la optimización de dicha bomba.

Energía solar, Bombeo solar, Panel solar

\begin{abstract}
The extraction of water from wells for supply in rural populations is one of the most profitable and novel applications of photovoltaic solar energy. The use of a specific type of submersible pumps for applications with photovoltaic energy, that work with direct / direct current (DC / DC) generated directly from the photovoltaic modules, allows an independent installation of the electrical network with very scarce maintenance needs, in This project was carried out the sizing, installation and commissioning of an autonomous pumping system with a submersible pump powered by a photovoltaic solar installation in the municipality of Actopan Hidalgo in the state of Pachuca. To implement the project, a climate study was first carried out. the area to support the efficiency of the solar pumping system, in this project we used 18 panels of $150 \mathrm{~W}$, a SubDrive Solar controller, a Franklin Electric pump of $3 \mathrm{HP}$ and a frequency inverter for the optimization of said pump.
\end{abstract}

Solar energy, Solar pump, Solar panel

Citación: FLORES-RAMIREZ, Oscar, HUESCA-LAZCANO, Erick Eduardo, ROMERO-RODRIGUEZ, Gabriel y JIMENEZ-SOTO Ezequiel. Sistemas de bombeo solar en Pachuca Hidalgo. Revista de Innovación Sistemática. 2019. 3-12: $17-27$

*Correspondencia al Autor (Correo electrónico: oscar.flores@upamozoc.edu.mx)

$\dagger$ Investigador contribuyendo como primer Autor. 


\section{Introducción}

La extracción de agua de pozos para suministro en poblaciones rurales es una de las aplicaciones más rentables y novedosa de la energía solar fotovoltaica. El empleo de un tipo de bombas sumergibles específicas para aplicaciones con energía fotovoltaica, que funcionan a corriente continua/directa (CC/DC) generada directamente de los módulos fotovoltaicos, posibilita una instalación independiente de la red eléctrica con muy escasas necesidades de mantenimiento.

Como se ha indicado, es necesario la instalación de unos paneles fotovoltaicos que producen energía eléctrica a una tensión de 12 o 24 voltios en corriente continua. Esta electricidad es consumida directamente por una bomba, también en corriente continua, que bombea el agua desde el fondo del pozo a un depósito con una cierta altura o directamente a la red de distribución de riego.

La falta de uniformidad derivada de la dependencia del rendimiento de los módulos fotovoltaicos a la intensidad de la radiación solar puede ser suplida por la instalación de un sistema de baterías de acumulación, de manera que la alimentación de corriente hacia la bomba se realice a través de ellas. Con ello se consigue una alimentación de corriente eléctrica constante que asegura un suministro uniforme de agua por parte de la bomba, independiente de las condiciones de radiación solar.

No obstante, otra alternativa a la anterior, y mucho más barata y simple, es la instalación de un sistema de bombeo solar directo, que no incluye baterías. En este caso, para conseguir la uniformidad en el riego se construye un depósito en altura de manera que el flujo de agua extraída por la bomba se dirija hacia este depósito de acumulación. Este depósito, que actuaría como depósito pulmón, permitiría realizar el riego de manera constante y uniforme, mientras el depósito disponga de un nivel de agua mínimo.

Por tanto, se tratará en este trabajo de describir los elementos que componen una instalación solar fotovoltaica autónoma para el bombeo directo de agua, incluyendo catálogos y hojas de especificaciones técnicas de los distintos equipos y exponer un caso práctico de cálculo, que pueda servir de guía y modelo para otras instalaciones.

\section{Planteamiento del problema}

Uno de los parámetros para medir la calidad de vida de una sociedad son los servicios con los que cuentan sus pobladores. Dentro de los cuales los principales servicios son el agua potable y la luz eléctrica. A pesar de la importancia de estos servicios muchas personas a nivel mundial no cuentan con alguno de ellos y en ocasiones con ninguno de los dos. Esta problemática es muy notoria en los países en desarrollo y se complica cuando la orografía del país es abrupta. Ambas condiciones se presentan en el caso de la República Mexicana, por lo que al año 2017 el $2.16 \%$ de su población no contaba con energía eléctrica, el $8.12 \%$ no tenía acceso a la red pública de distribución de agua y el 1.68\% carecía de ambos servicios.

El agua es un recurso necesario para el desarrollo y para la vida del hombre. Desgraciadamente hay muchos lugares en el mundo donde el acceso al vital líquido es complicado, en ocasiones debido a la falta del mismo, pero en otros casos debido a la dificultad para trasladarlo del lugar en que se encuentra (lagos, ríos, pozos o redes de distribución) a las zonas donde se necesita. En este último caso, los habitantes de estos lugares se ven obligados a ir a la fuente de agua y acarrear el agua hasta sus hogares, tarea que la mayoría de las veces se realiza a pie. Justificación

Un hecho real es el cambio climático, que se está generando por el consumo energético de los combustibles fósiles (petróleo, carbón y gas), sin darnos cuenta de que este consumo energético es insostenible, debido a que los yacimientos de combustibles se están agotando; adicionalmente está ocasionando graves trastornos ambientales deteriorando el planeta. para nadie es un secreto que el calentamiento global es un problema actual y a nivel mundial algunas naciones empiezan a cambiar sus formas de producción y sus normas con el fin de tratar de disminuir los efectos de su industria en el ambiente, así mismo se buscan formas de producción de energía alternativas, pues es ya sabido que el gran problema gira entorno a la liberación de bióxido de carbono $\left(\mathrm{CO}_{2}\right)$ producido por la quema de esos combustibles que son generados por los medios de transporte, los electrodomésticos y las industrias. 


\section{Objetivo general}

Dimensionar y poner en marcha un sistema de bombeo solar fotovoltaico para el municipio de Actopan, Pachuca Hidalgo.

\section{Objetivos específicos}

- Se dimensionará el sistema
fotovoltaico.
Se calculará la bomba para
abastecimiento de la comunidad de
Pachuca Hidalgo.
Se instalará el sistema de bombeo
fotovoltaico.

\section{Marco Teórico}

Ahora bien, la pregunta que nos debemos plantear es ¿Qué podemos obtener con la energía solar? para obtener la respuesta es necesario saber cuáles son los efectos que puede producir la energía solar, hechas las consultas pertinentes tenemos que el calor se logra mediante un mecanismo de captación realizado por los colectores térmicos y la electricidad es producida por medio de los módulos fotovoltaicos, que puede ser usada en el uso de electrodomésticos.

Los paneles solares se perfilan como la solución definitiva al problema de electrificación rural, debido a las largas distancias para enviar energía, se hace más fácil el mantenimiento, no produce ruido y lo mejor no consume combustible; una verdadera ventaja es que funciona también en días nublados ya que capta la luz que se filtra en las nubes y ayuda a la conservación del medio ambiente.

\section{Sistema de bombeo solar}

Una instalación solar fotovoltaica para bombeo directo de agua está destinada a satisfacer las necesidades de consumo propio de electricidad para el accionamiento de la bomba, y este está compuesto por un arreglo de módulos FV, un controlador, un motor y una bomba. Se emplean motores de corriente alterna (CA) y de corriente continua (CC). Las bombas pueden ser centrífugas o volumétricas. Generalmente el agua se almacena en un tanque, en la figura 1 se muestra el esquema de un sistema de bombeo solar.

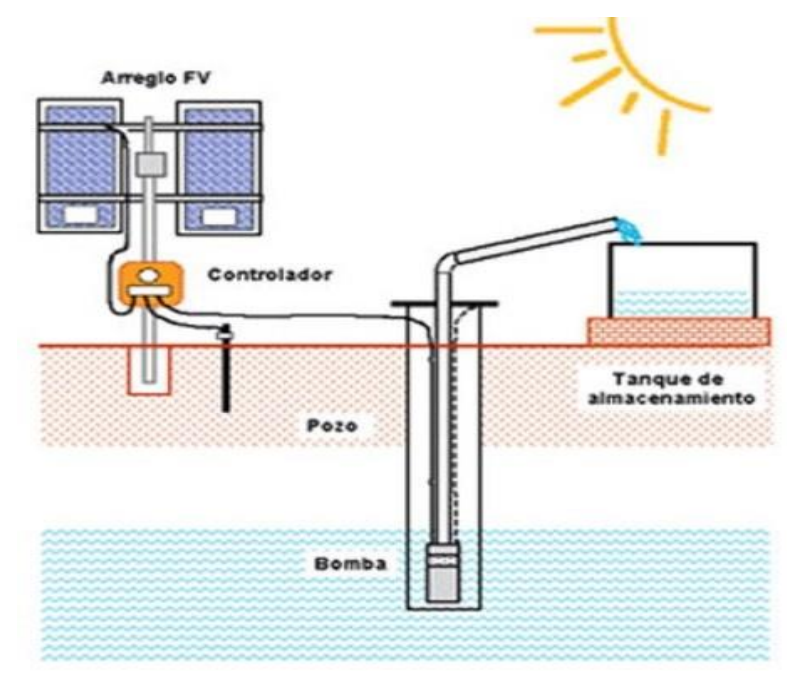

Figura 1 Esquema de un sistema de bombeo solar Fuente: Luminasol.com.mx

\section{Paneles o módulos solares}

Son los encargados de captar la radiación solar y transformarla en electricidad, generando una corriente continua (CC), también llamada corriente directa (DC) que alimenta a la bomba. El número de paneles quedará determinado por la potencia que se necesita suministrar a la bomba, de acuerdo con el caudal de agua a bombear y presión de suministro. Asimismo, la disposición y forma de conexión de los paneles (en serie o en paralelo), será en función de la tensión nominal y la intensidad de corriente necesaria para el accionamiento del motor eléctrico de la bomba.

Los paneles solares se situarán sobre un rack o estructura metálica a cierta altura para evitar que se proyecten sombras sobre la superficie de los paneles debida a la presencia de árboles o de cualquier otro obstáculo cercano.

\section{Regulador o controlador de carga}

Dispositivo electrónico encargado de controlar el funcionamiento óptimo de la bomba de agua. El controlador ayuda a maximizar el rendimiento energético de los paneles solares, permitiendo que la bomba de agua funcione también durante periodos de menor irradiación solar. El controlador además regula el funcionamiento de la bomba, desconectándola cuando el depósito donde se bombea el agua haya llegado a su capacidad máxima o bien, porque el nivel del agua en el pozo haya bajado de un límite de seguridad establecido, con el fin de evitar que se quede descubierta la boca de aspiración de la bomba. 
Asimismo, el regulador de carga dispone de un sistema de control con conectores "Plug\&Play" de posición única, que permite el encendido o apagado del sistema (en invierno, por ejemplo, como es época de lluvias se desconecta, situando el interruptor en posición "Off").

Las bombas comunes disponibles en el mercado han sido desarrolladas pensando en que hay una fuente de potencia constante. Por otro lado, la potencia que producen los módulos FV es directamente proporcional a la disponibilidad de

la radiación solar. Es decir, a medida que el sol cambia su posición durante el día, la potencia generada por los módulos varía y en consecuencia la potencia entregada a la bomba. Por esta razón se han diseñado algunas bombas especiales para la electricidad fotovoltaica las cuales se dividen, desde el punto de vista mecánico, en centrífugas y volumétricas.

\section{Bombas centrífugas}

Tienen un impulsor que por medio de la fuerza centrífuga de su alta velocidad arrastran agua por su eje y la expulsan radialmente. Estas bombas pueden ser sumergibles o de superficie y son capaces de bombear el agua a 60 metros de carga dinámica total, o más, dependiendo del número y tipo de impulsores. Están optimizadas para un rango estrecho de cargas dinámicas totales y la salida de agua se incrementa con su velocidad rotacional.

Las bombas de succión superficial se instalan a nivel del suelo y tienen la ventaja de que se les puede inspeccionar y dar servicio fácilmente. Tienen la limitante de que no trabajan adecuadamente si la profundidad de succión excede los 8 metros.

Hay una gran variedad de bombas centrifugas sumergibles. Algunas de estas bombas tienen el motor acoplado directamente a los impulsores y se sumergen completamente. Otras, tienen el motor en la superficie mientras que los impulsores se encuentran completamente sumergidos y unidos por una flecha. Generalmente las bombas centrífugas sumergibles tienen varios impulsores y por ello, se les conoce como bombas de paso múltiple o de etapas. En la figura 2 se muestra un esquema de bomba centrífuga sumergible.

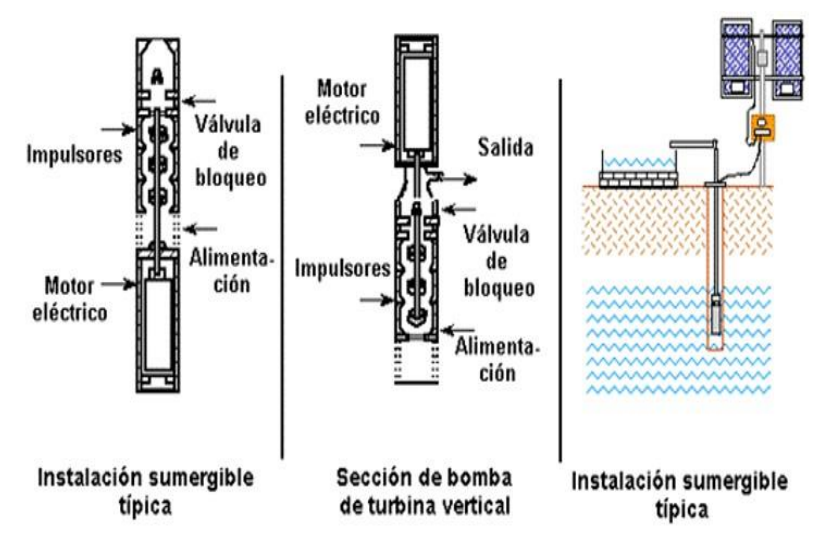

Figura 2 Esquema de una bomba centrífuga sumergible Fuente: Luminasol.com.mx

\section{Tipos de motores}

La selección de un motor depende de la eficiencia, disponibilidad, confiabilidad, acoplamiento a bombas y costos. Comúnmente se usan dos tipos de motores en aplicaciones FV: De CC (de imán permanente y de bobina) y de corriente alterna CA. Debido a que los arreglos $\mathrm{FV}$ proporcionan potencia en $\mathrm{CC}$, los motores de $\mathrm{CC}$ pueden conectarse directamente, mientras que los motores de $\mathrm{CA}$ deben incorporar un inversor CC/CA. Los requerimientos de potencia en Watts pueden usarse como una guía general para la selección del motor.

Los motores de CC de imán permanente, aunque requieren reemplazo periódico de las escobillas, son sencillos y eficientes para cargas pequeñas. Los motores de CC de campos bobinados ( $\sin$ escobillas) se utilizan en aplicaciones de mayor capacidad y requieren de poco mantenimiento. Aunque son motores sin escobillas, el mecanismo electrónico que sustituye a las escobillas puede significar un gasto adicional y un riesgo de descompostura.

Los motores de CA son más adecuados para cargas grandes en el rango de diez o más caballos de fuerza. Los sistemas de CA son ligeramente menos eficientes que los sistemas CC debido a las pérdidas de conversión. Los motores de CA pueden funcionar por muchos años con menos mantenimiento que los motores CC. En la tabla 1, se muestran las ventajas y desventajas del bombeo solar. 


\begin{tabular}{|l|l|}
\hline \multicolumn{1}{|c|}{ Ventajas } & \multicolumn{1}{|c|}{ Desventajas } \\
\hline $\begin{array}{l}\text { No consumen } \\
\text { combustible }\end{array}$ & Inversión inicial relativamente \\
Larga vida útil (de 15 & Acceso a servicio técnico \\
a 20 años) ambiental & limitado \\
Impacto & $\begin{array}{l}\text { Producción de agua variable } \\
\text { mínimo dependiendo de condiciones } \\
\text { Bajos costos de } \\
\text { operación y } \\
\text { Mantenimiento }\end{array}$ \\
\hline
\end{tabular}

Tabla 1 Ventajas y desventajas del bombeo solar Fuente: Elaboración Propia

\section{Metodologìa}

\section{Descripción del método}

El dimensionado de sistemas de bombeo fotovoltaico se puede abordar mediante diferentes métodos, dependiendo su complicación, del número de variables consideradas y del grado de optimización en la obtención de los resultados.

Para la realización de este proyecto el método consistió en los siguientes pasos:

- Se realizó un estudio de la zona en donde se implementó el sistema de bombeo solar, obteniendo datos como la radiación incidente, latitud, longitud, etc. Se evaluó de la energía hidráulica necesaria.

Se obtuvo disponibilidad del recurso solar, es decir, qué tanta energía solar hay en la región geográfica.

Se calculó el caudal diario que deseamos suministrar $\left(\mathrm{m}^{3} /\right.$ día$)$.

Se obtuvo la altura total de bombeo (altura manométrica) en metros.

Se determinó el periodo de funcionamiento (si es anual o estacional). Posteriormente se calculó el número de paneles necesarios para abastecer la demanda energética de la bomba, también el número de paneles que deben estar conectados en serie y en paralelo, se determinó el tipo de estructura a utilizar para montar los paneles solares y finalmente, la inclinación optima que estos deben tener respecto a la latitud del lugar.

- Se determino el controlador a utilizar para satisfacer las necesidades de la bomba solar.
Posteriormente calculamos el cableado ideal para nuestra instalación, en el cual se debe prestar mucha atención, puesto que, al pasar energía por nuestros cables, siempre existirán perdidas que se deben a las caídas de tensión que hay en ellos.

- Realizamos la puesta en marcha del sistema de bombeo solar.

\section{Resultados}

Los datos del sistema de bombeo solar se muestran en la tabla 2 .

$\begin{gathered}\text { El sistema instalado en Pachuca Hidalgo se diseñó con } \\
\text { los siguientes datos: }\end{gathered}$
\begin{tabular}{|l|r|} 
Nivel estático del agua & $34 \mathrm{~m}$ \\
\hline Abatimiento & $5 \mathrm{~m}$ \\
\hline Altura de la descarga & $10 \mathrm{~m}$ \\
\hline Distancia al depósito: & $8 \mathrm{~m}$ \\
\hline Requerimiento diario: & $15000 \mathrm{l} / \mathrm{d}$ dí \\
\hline
\end{tabular}

Tabla 2 Datos para el sistema de bombeo solar. Fuente: Elaboración Propia

Para calcular la bomba que cumpla con los requisitos solicitados, primero se obtuvo la carga dinámica total (CDT), que es la suma de la carga estática $(\mathrm{CE})$ más la carga dinámica $(\mathrm{CD})$.

Obtenemos $\mathrm{CE}=$ Nivel estático + Abatimiento + Altura de la descarga $=49 \mathrm{~m}$.

Para obtener CD, se utilizó la fórmula de Manning, sabiendo que se utilizaría material PVC por ser económico y durable, esto nos sirvió para saber el valor de $\mathrm{k}$, de los datos del sistema se sabe que el volumen es de15000 litros equivalente a $15 \mathrm{~m}^{3}$ y por tanto $\mathrm{Q}=7.75 \times 10^{-4}$ $\mathrm{m}^{3} / \mathrm{s}$.

Así tenemos que:

$\mathrm{CD}=\kappa \times \mathrm{L} \times \mathrm{Q}^{2}=31,282(\mathrm{~m} 3 / \mathrm{s})^{-2} \times 57 \mathrm{~m} \times$ $\left(7.75 \times 10^{-4} \mathrm{~m}^{3} / \mathrm{s}\right)^{2}=1.07 \mathrm{~m}$

La Carga Dinámica Total es entonces

$\mathrm{CDT}=\mathrm{CE}+\mathrm{CD}=49 \mathrm{~m}+1.07 \mathrm{~m}=50.07 \mathrm{~m}$

En la figura 3 se muestra la captura de los datos de CDT y caudal en el software de Franklin Electric, para obtener el tipo de bomba y el tipo de controlador. 


\section{APLICACIÓN Sumergible •}

\section{COND. BÁSICAS DE SERVICIO}

$\begin{array}{lll}\text { Altura manométrica } & 50.00 & \mathrm{~m} \\ \text { Consigna } & \text { Caudal } \\ \text { Caudal } & & \\ \text { Tipo de rosca } & \text { Según ubicación } \\ \text { Catálogo regional } & \text { Segun ubicacion } \\ \text { *Temp. Máx. admisible de } 30^{\circ} \mathrm{C} / 86^{\circ} \mathrm{F} \text {. Consulte a Franklin } \\ \text { para valores más altos }\end{array}$

Figura 3 Captura de datos del sistema de bombeo solar en el software de Franklin Electric

Fuente: solar.franklin-electric.com/

En la figura 4 se muestra la captura de los datos de latitud y longitud del lugar donde se instalará el sistema de bombeo solar en el software de Franklin Electric, para obtener el tipo de bomba y el tipo de controlador.

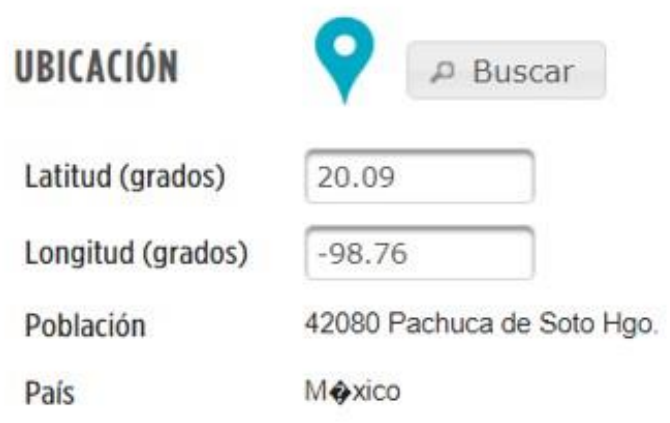

Figura 4 Captura de datos del sistema de bombeo solar en el software de Franklin Electric

Fuente: solar.franklin-electric.com/

En la figura 5 se muestra la captura de los datos del panel solar y del cable utilizar del sistema de bombeo solar en el software de Franklin Electric.

\section{CARACTERISIICAS PANEL SOLAR}

Condiciones de Servicio
Watts (Wmpp)
Volts (Vmpp)
Volts (Voc)
Seguidor solar
Mes Máximo
CABLE*
Longitud
Material
Temperatura aislamiento
$\begin{aligned} & \text { *El dimensionamiento y longitudes de cable deben cumplir } \\ & \text { con la normativa local, nacional o normas eléctricas } \\ & \text { vigentes aplicables. }\end{aligned}$

Figura 5 Captura de datos del sistema de bombeo solar en el software de Franklin Electric

Fuente: solar.franklin-electric.com/

En la gráfica 1 se muestra el promedio mensual de la irradiación solar de Pachuca Hidalgo y el promedio anual utilizado para calcular el sistema fotovoltaico que abastece de energía a la bomba.

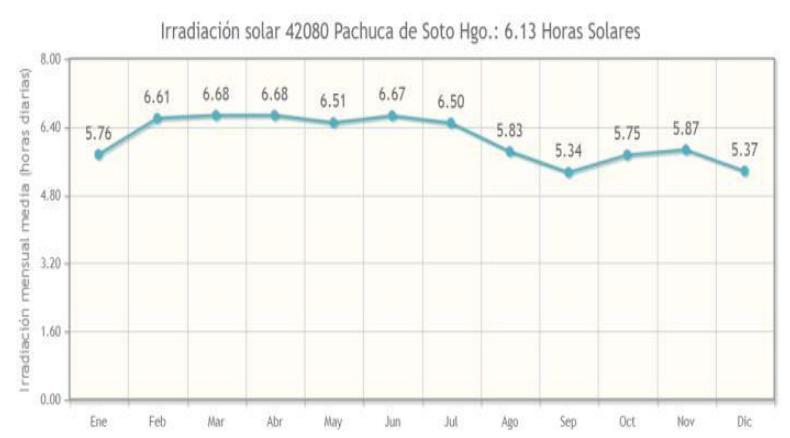

Gráfica 1 Se muestra el promedio mensual y anual de la irradiación solar de Pachuca Hidalgo Fuente: solar.franklin-electric.com/

En la figura 6 se muestran los resultados obtenidos de los modelos de bombas que cumplen con los datos del sistema de bombeo solar, de igual forma se observa el número de paneles necesarios para el buen funcionamiento de la bomba, la media diaria de bombeo 


\begin{tabular}{|c|c|c|c|c|c|c|c|c|c|c|c|}
\hline 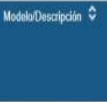 & 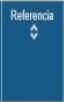 & $\begin{array}{l}\text { Potenda } \\
\text { Etrota } \\
\hat{v}\end{array}$ & 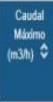 & 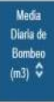 & $\begin{array}{l}\text { Vurnamad } \\
(m) \hat{v}\end{array}$ & 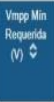 & 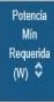 & 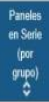 & $\begin{array}{l}\text { Ninte } \\
\text { Ganpe } \\
i s\end{array}$ & 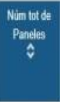 & 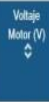 \\
\hline 15SDSP. $0.75 \mathrm{HP}$ & 90041510 & $D C A C$ & 2.87 & 17.59 & 0.01 & 144 & 1,152 & 10 & 1 & 10 & 100 \\
\hline 15FDSP. $075 \mathrm{HP}$ & 90041514 & DC & 2.93 & 17.96 & 0.01 & 144 & 1,128 & 10 & 1 & 10 & 100 \\
\hline 25SDSPP-1.5HP & 90042520 & DCAC & 3.59 & 22.01 & 0.01 & 217 & 961 & 13 & 1 & 13 & 200 \\
\hline 25FDSP-1.5HP & 90042524 & DC & 3.59 & 22.01 & 0.01 & 217 & 942 & 13 & 1 & 13 & 200 \\
\hline 15SDSP-1.5HP & 9004520 & $D C A C$ & 296 & 18.14 & 0.01 & 220 & 1,147 & 13 & 1 & 13 & 200 \\
\hline 15FDSP-1.5HP & 90041524 & DC & 2.96 & 18.14 & 0.01 & 220 & 1,123 & 13 & 1 & 13 & 200 \\
\hline 10SOSP $1.5 \mathrm{HP}$ & 90041020 & $D C A C$ & 2.84 & 17.41 & 0.01 & 257 & 1,683 & 15 & 1 & 15 & 200 \\
\hline 10FDSP-1.5HP & 90041024 & DC & 284 & 17.41 & 0.01 & 257 & 1,649 & 15 & 1 & 15 & 200 \\
\hline 25SDSP-3.OHP & 90042530 & $D C A C$ & 3.92 & 24.03 & 0.02 & 213 & 976 & 13 & 1 & 13 & 200 \\
\hline 1550SP-3.0HP & 90041530 & DCAC & 3.11 & 19.06 & 0.01 & 214 & 1,167 & 13 & 1 & 13 & 200 \\
\hline 10SDSP-3.0HP & 90041030 & DCAC & 275 & 16.86 & 0.01 & 247 & 1,784 & 15 & 1 & 15 & 200 \\
\hline 75DSP-3.0HP & 90040730 & DCAC & 2.90 & 17.78 & 0.00 & 283 & 2,379 & 17 & 2 & 34 & 200 \\
\hline $\begin{array}{c}\text { F3SP45HP230+ } \\
\text { 10FASS4PE }\end{array}$ & $\begin{array}{c}3071212002 \\
+ \\
932210035\end{array}$ & DC & 275 & 16.86 & 0.01 & 281 & 2,713 & 23 & 1 & 23 & 230 \\
\hline
\end{tabular}

Figura 6 Resultados obtenidos del software de Franklin Electric

Fuente: solar.franklin-electric.com/

En la figura 7 se muestra el modelo de la bomba a utilizar y los requerimientos mínimos de voltaje y potencia para su buen funcionamiento.

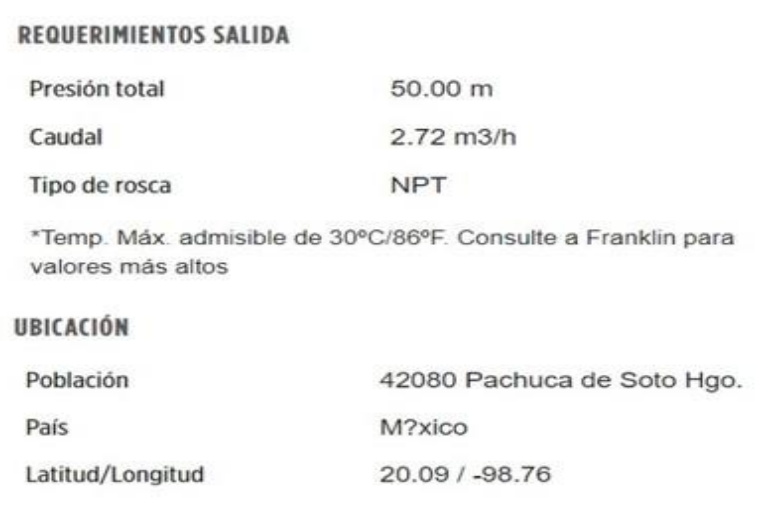

\section{REQUERIMIENTOS MÍNIMOS}

$\begin{array}{ll}\text { Vmpp } & 217 \mathrm{~V} \\ \text { Wmpp } & 961 \mathrm{~W} \\ \text { CONFIG. } & \\ \text { Modelo } & 25 S \mathrm{SSP}-1.5 \mathrm{HP} \\ \text { Código modelo } & 90042520 \\ \text { Caudal máximo } & 3.59 \mathrm{~m} 3 / \mathrm{h}\end{array}$

Figura 7 Modelo de bomba solar utilizado Fuente: solar.franklin-electric.com/

En la figura 8 se muestran las características del panel solar utilizado para satisfacer las necesidades energéticas de la bomba solar, el ángulo al que deben colocarse los paneles y el calibre del cable utilizado.

\section{CARACTERÍSTICAS DEL PANEL}

$\begin{array}{ll}\text { Condiciones de Servicio } & \text { STC } \\ \text { Uso de seguidor solar } & \text { No } \\ \text { Watts (Wmpp) } & 150 \mathrm{~W} \\ \text { Volts (Vmpp) } & 19.0 \mathrm{~V} \\ \begin{array}{l}\text { Volts (Voc) } \\ \text { Ánngulo de inclinación } \\ \text { recomendado (grados) }\end{array} & 23.0 \mathrm{~V} \\ \text { CABLEAD0* } & 20.09 \\ \text { Sección cable - US (métrico) } & 14\left(2.5 \mathrm{~mm}^{2}\right)\end{array}$

Figura 8 Características del panel solar, ángulo de inclinación y calibre del cable.

Fuente: solar.franklin-electric.com/

En la figura 9 se muestra la configuración del sistema fotovoltaico para satisfacer las necesidades energéticas de la bomba solar, se observa que hay una cadena de 13 paneles en serie

$\begin{array}{ll}\text { CONFIG. INST. SOLAR } & \\ \text { Paneles en serie } & 13 \\ \text { Número de grupos } & 1 \\ \text { Núm. total paneles } & 13 \\ \text { Potencia inst. (Wmpp) } & 1,560 \mathrm{~W} \\ \text { Tensión inst. (Vmpp) } & 230 \mathrm{~V} \\ \text { Tensión inst. (Voc) } & 284 \mathrm{~V}\end{array}$

Figura 9 Configuración del sistema fotovoltaico Fuente: solar.franklin-electric.com/

En la gráfica 2 se muestra el promedio mensual del volumen generado por el sistema de bombeo solar, el agua es almacenado en un tanque para su uso en horas sin sol.

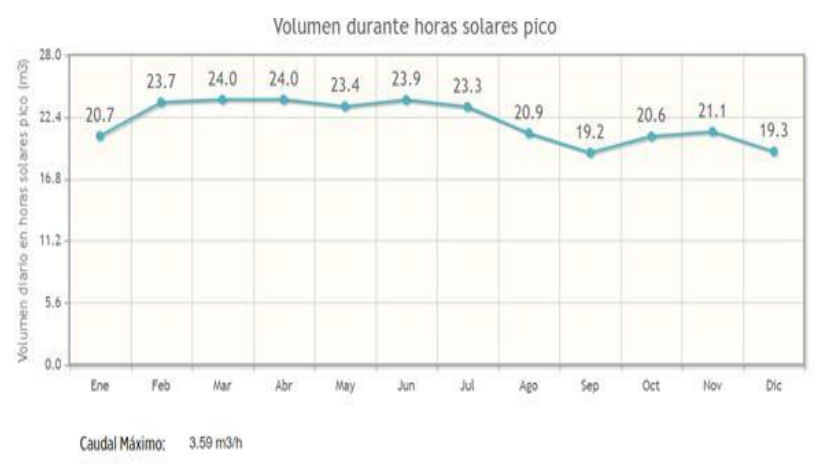

Gráfica 2 Promedio mensual del volumen generado por el sistema de bombeo solar Fuente: solar.franklin-electric.com/ 
En la figura 10 se muestra el tipo de controlador a que se utilizó para satisfacer las necesidades de la bomba solar.

\section{ESPECIFICACIONES CONTROLADOR SOLAR SUBDRIVE

$\begin{array}{ll}\text { Modelo } & 5870301113 \\ \text { Tamaño } & 533 \mathrm{~mm} \times 259 \mathrm{~mm} \times 219 \mathrm{~mm} \\ \text { Peso } & 18.60 \mathrm{~kg} \\ \text { Energia Alimentacion } & \text { DC/AC }\end{array}$

\section{SALIDA}

$\begin{array}{ll}\text { Máx Tensión salida } & \text { 200V AC, 3-fase } \\ \text { Máx. intensidad (RMS) } & 6.8 \mathrm{~A}, \text { por fase } \\ \text { Frecuencia salida } & 20-60 \mathrm{~Hz} \\ \text { Eficiencia a pot. máx. } & 96 \%\end{array}$

\section{INST. FOTOVOLTAICA}

\section{Tensión alim. mpp \\ Máx. intensidad alim.}

Potencia en mpp

\section{CONDICIONES DE SERVICIO}

\section{Temperatura}

Humedad Relativa $-25^{\circ} \mathrm{C}$ a $50^{\circ} \mathrm{C}$ (con generador de $\mathrm{CA}$, máx $40^{\circ} \mathrm{C}$ )

$$
\text { o a } 100 \% \text { (Condensación) }
$$

Figura 10 Datos del controlador Fuente: solar.franklin-electric.com/

En la figura 11 se muestra el retorno de inversión del sistema de bombeo solar para Pachuca Hidalgo.

\begin{tabular}{|c|c|c|c|c|}
\hline \multicolumn{5}{|c|}{ "ENTER DATA IN THE GREY BOXES TO CALCULATE CORRECTLY } \\
\hline \multicolumn{5}{|c|}{ *PLEASE ADJUST TOTAL SYSTEM COST TO BEGIN CALCUATIONS } \\
\hline \multicolumn{2}{|c|}{ Initial Investment } & \multicolumn{3}{|c|}{ Powered by the Grid } \\
\hline Total System Cost & $\$ 4,000.00$ & Motor Power Draw (Watts) & 1500 & \\
\hline & & Hours Used/Day & 7 & \\
\hline \multicolumn{2}{|c|}{ Solar Rebates } & Watt-Hours Usage/Day & & $10.50 \mathrm{KWh} / \mathrm{day}$ \\
\hline Tax credit (\%) & $30 \%$ & Days Used/Month & 30.5 & \\
\hline Subsidies (\$) & $\$$ & Power Requirement/Month & & $320.25 \mathrm{kWh}$ \\
\hline Add items (\$) & $\$$ & Cents/kWh & 20 & \\
\hline Add items (\$) & $\$$ & Annual Increases (\%) & $3 \%$ & \\
\hline Net Investment Cost & 2,800 & Monthly Savings & & 65.97 \\
\hline
\end{tabular}

\begin{tabular}{|r|r|}
\hline \multicolumn{2}{|c|}{ Return On Investment } \\
\hline Return on Inv. (Months) & 42.44 \\
\hline Return on Inv. (Years) & 3.54 \\
\hline
\end{tabular}

Figura 11 Retorno de inversión del sistema Fuente: solar.franklin-electric.com/

En este proyecto se utilizaron módulos solares fijos montados sobre una estructura metálica colocado a cierta altura sobre un mástil o poste también metálico con el fin de evitar que se proyecten sombras sobre la superficie de los paneles debida a la presencia de árboles o de cualquier otro obstáculo cercano, los paneles se orientaron hacia el sur con un ángulo de inclinación de $20^{\circ}$ para garantizar la máxima producción de energía eléctrica media anual, como se observan en las figuras 12 y 13.

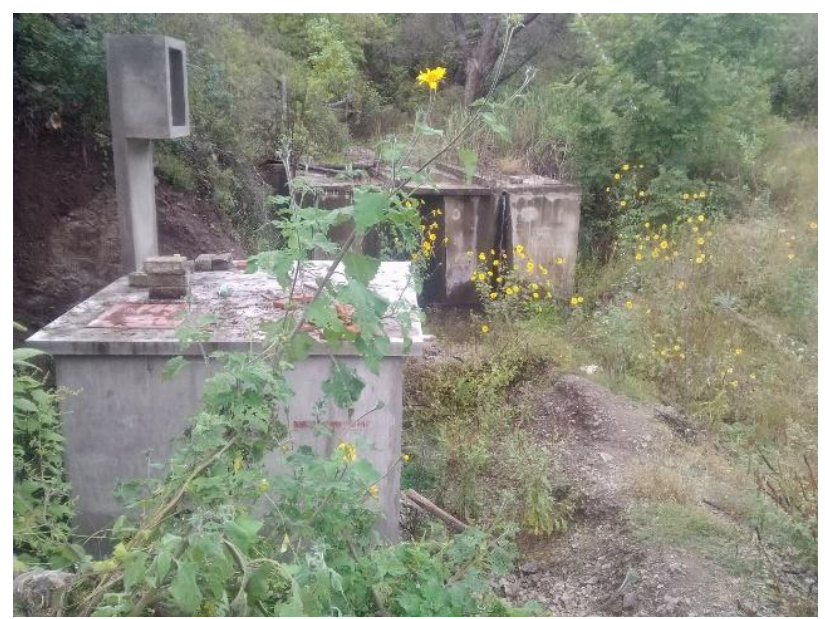

Figuras 12 Pozo en Pachuca Hidalgo Fuente: Elaboración Propio 


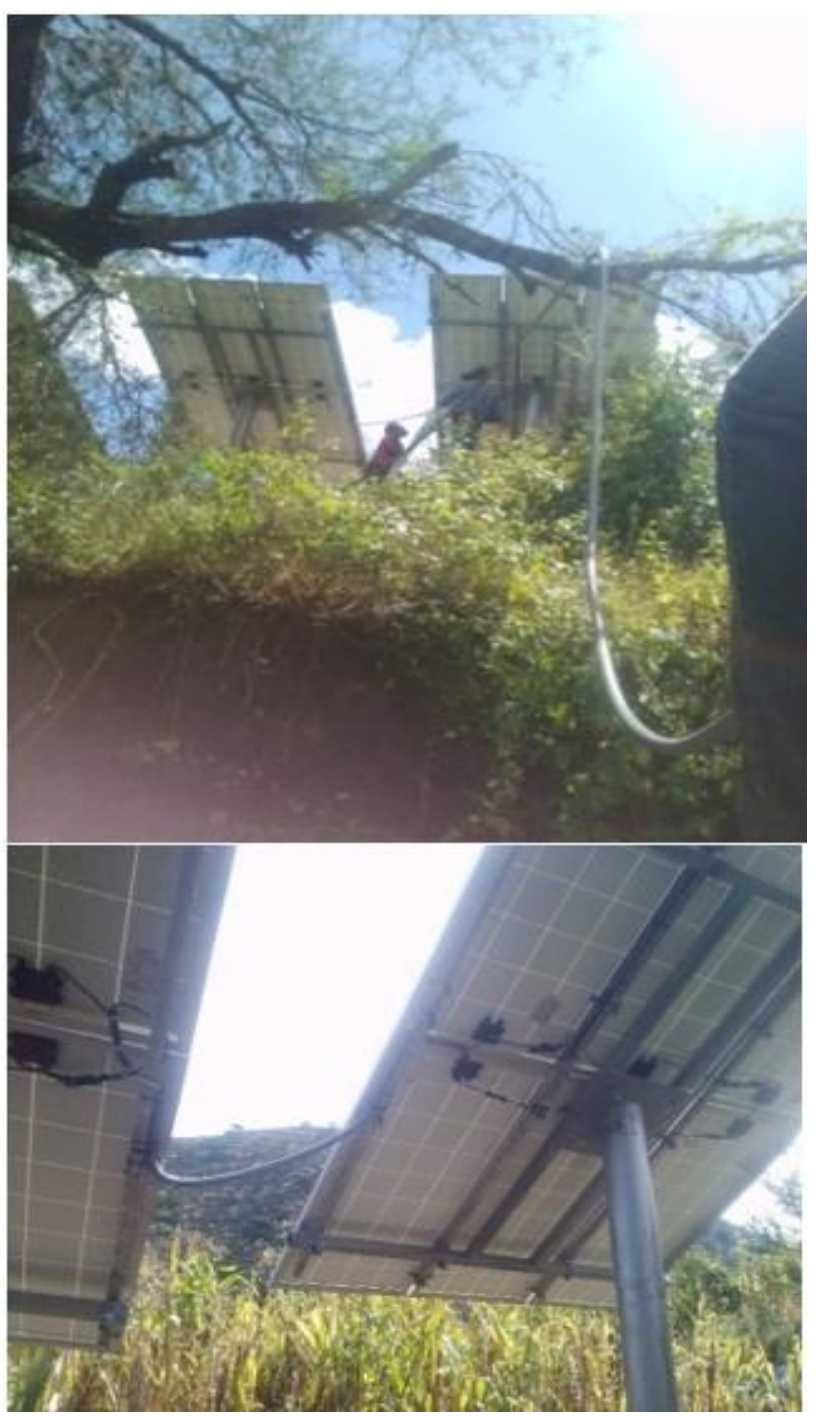

Figuras 13 Instalación de del sistema fotovoltaico Fuente: Elaboración Propio

\section{Agradecimiento}

A la Universidad Politécnica de Amozoc por el "Apoyo a la Investigación Científica y Desarrollo Tecnológico" y por gestionar los recursos ante las instancias correspondientes para el financiamiento del proyecto.

\section{Conclusiones}

Se realizó el estudio del recurso solar de Pachuca Hidalgo obteniendo un promedio anual de 6.13 horas solares con este dato se pudo obtener el dimensionamiento del sistema de bombeo solar. Se calculo la carga dinámica, la carga estática y la carga dinámica total para poder obtener el modelo de la bomba y el modelo del controlador. $\mathrm{Se}$ dimensiono el sistema fotovoltaico obteniéndose 13 paneles conectados en serie, llamada cadena con un voltaje de $247 \mathrm{~V}$ y una corriente de 7A, suficiente para que la bomba solar funcione adecuadamente, los paneles se montaron en estructuras con monopostes por economía.
Se calculó la bomba solar mediante el software de la empresa Franklin Electric, el modelo obtenido fue 25SDSP-1.5HP, capas impulsar $3.59 \mathrm{~m}^{3} / \mathrm{h}$, mayor a los requerimientos solicitados, pero se tomó la decisión con el cliente de colocar esta bomba por el crecimiento poblacional.

El modelo del controlador solar obtenido fue el 5870301113 con energía de alimentación DC/AC, con una tensión de salida de $200 \mathrm{~V}$ AC, 3 -fases y corriente RMS de $6.8 \mathrm{~A}$.

Se instaló el sistema de bombeo solar en Pachuca Hidalgo y monitoreo durante el mes de abril obteniendo un volumen de agua impulsada al contenedor de $23.1 \mathrm{~m}^{3} / \mathrm{mes}$ promedio, comparando con los datos obtenidos del software tenemos un margen de error de 4 $\%$. El retorno de inversión está calculado para aproximadamente 4 años y el sistema tiene una funcionalidad de 20 años.

\section{Recomendaciones}

1. Respetar la normativa y reglamentos electrotécnicos aplicables a instalaciones eléctricas.

2. Verificación del pozo de agua (capacidad de producción) u obra civil: cimientos, tuberías y depósito de almacenamiento.

3. Proteger la entrada de la bomba de la posible entrada de suciedad o arena mediante filtros adecuados en cada caso. Tener en cuenta las posibles pérdidas de carga de estos filtros.

4. Evitar tuberías muy largas. Las pérdidas de carga pueden aumentar considerablemente la altura total de bombeo y por tanto el tamaño del generador FV.

5. Proteger de la intemperie los equipos electrónicos en cajas que cumplan con la protección IP65. El cableado de la instalación debe cumplir con la normativa eléctrica vigente, minimizando las caídas de tensión. Los cables utilizados para bombas sumergibles deben ser apropiados para tal fin. 


\section{Referencias}

Almanza-Fundora, I., García-Fernández, R. R., \& García-Reina, F. (2019). Desarrollo de un sistema para la medición de la eficiencia energética de celdas solares. Revista Ciencias Técnicas Agropecuarias, 28(1).

Anguiano J. (2012). Constante solar. P.15 - 17 Impacto de la temperatura de los módulos en la eficiencia de un sistema fotovoltaico conectado a la red. Recuperado de: http://148.206.53.84/tesiuami/UAMI16030.pdf

Barrow R. McNeil B., Erick A. (1993) Solar Pumping: An Introduction and Update on the Technology, Performance, Costs and Economics (Bombeo Solar: Introducción y Actualización de Tecnología, Desempeño y Costos). World Bank Intermediate Technology Publications. Artículo No 168, Washington, D.C.

Cabanillas, R.E., Munguía, H. (2011). Dust accumulation effect on efficiency of $\mathrm{Si}$ photovoltaic modules. Journal of Renewable and Sustainable Energy, 3.

Canarias, G. D. (2018). Instalaciones fotovoltaicas. Obtenido de http://www.agenergia.org/files/resourcesmodul e/@random49914e4ed9045/1234263307_Guia FotovoltaicaGobCan.pdf

Castilla, J. (2007). Introducción a la energía solar fotovoltaica. Pp: 15-16. Manual del instalador energía fotovoltaica. Gráficas Celarayn, España

Daniel Almarza, A. G. (marzo de 2017). Guía de buenas y malas prácticas de instalaciones fotovoltaicas sobre techos. Documento anexo a la. Obtenido de http://www.sec.cl/pls/portal/docs/PAGE/SEC20 05/ELECTRICIDAD_SEC/ERNC/GENERACI ON_DISTRIBUIDA/LINKS_Y_NOTICIAS/T AB6243717/GU\%CDA-DE-BUENAS-YMALAS-PR\%C1CTICAS-DEINSTALACIONES-FOTOVOLTAICAS..PDF

Domínguez H. (2012). Diseño de un Sistema fotovoltaico. Recuperado de: http://cdigital.uv.mx/bitstream/1234567893156 11dominguezgonzalezhector.pdf
Fletes, N., Paredes, C., Dibene, L. (2016). Eficiencia en la producción de energía de un panel fotovoltaico a diferente inclinación en Nuevo Vallarta, Nayarit. Revista de Aplicaciones de Ingeniería, 3.

Flores, O., González, R., Juárez, V., Huitzil, I. (2016). Análisis comparativo de rendimiento, costo y producción energética entre el sistema aislado e interconectado de la empresa cartón corrugado Puebla S.A. de C.V. Revista de Aplicaciones de Ingeniería, 3(9).

Flores, O., Huitzil, I., González, R. y Hernández, A. (2017). Reducción de la eficiencia energética del parque solar UPAM por suciedad y deposición de polvo. Revista de Innovación Sistemática, 1(3), 26-36

Flores, O., Huesca, E., Pacheco, E.A. y Aguilar, J.M. (2018). Dimensionamiento, instalación y puesta en marcha del Parque Solar UPAM de 50 $\mathrm{kW}$ de potencia. Revista de Energías Renovables, 2(5), 1-13

Julca Orozco, T. E. (2019). Diseño de un sistema de bombeo con energía solar para la localidad Hispon distrito La Matanza-Morropón-Piura.

Liqun, L., Zhiqi, L., Chunxia, S.Z.L. (2012). Degraded output characteristic at atmospheric air pollution and economy analysis of PV power system: a case study. Przegl. Elektrotech. (Electr. Rev.) 88 (9A), pp. 281-284.

Lobato, G. (s.f.). SUNCRAFT. Obtenido de SUNCRAFT: http://www.suncraft.com.mx/ Cerdan Cabrera, A. M. (2011). Diseño de un sistema de bombeo solar-eólico. en a. m. cabrera, diseño de un sistema de bombeo solareólico (pág. 126). Veracruz: Universidad Internacional de Andalucia.

Martínez, B., \& Carlos, J. (2019). Potencial De Un Sistema De Refrigeración Solar Por Absorción En Zonas De Alta Irradiación En Colombia Para Cargas De Refrigeración Médicas Y Conservación De Alimentos.

Mertens, K. (2014). Photovoltaics Fundamentals, Technology and Practice). United Kingdom: John Wil ey \& Sons Ltd

Ramírez E. (2011). Proyecto Ahorro de Energía. Recuperado 20/07/2014 del sitio web http://cdigital.uv.mx/bitstream/123456789/3305 4/1/ramirezrojas.pdf 
Rua, E., Gonzalez, A., Granados, A., \& Ramírez, R. (2019). Diseño estructural de transporte para sistema de bombeo portátil activado con energía solar fotovoltaica para el departamento de boyacá. revista ambiental agua, aire y suelo, 9(2).

Vera, C., \& Jarol, L. (2019). Dimensionamiento de un sistema de bombeo fotovoltaico para suministrar agua en el caserío Chillvaca en el distrito de Incahuasi-Ferreñafe Lambayeque.

Zorrilla, J., Piliougine, M., Carretero, J. Bernaola, P., Carpena, P., Mora. L., Cardona, M. (2011). Analysis of Dust Losses in Photovoltaic Modules. Congreso Mundial de Energías Renovables, Linkpoing, Suecia. 